\title{
A New Algebraization of the Lamé Equation*
}

\author{
FEDERICO FINKEL ${ }^{\dagger}$ \\ Department of Mathematics \\ Imperial College \\ London SW7 2BZ, UK
}

\begin{abstract}
Artemio GonzÁlez-López, Miguel A. Rodríguez
Departamento de Fúsica Teórica II

Universidad Complutense de Madrid

28040 Madrid, SPAIN
\end{abstract}

July 29, 1999

\begin{abstract}
We develop a new way of writing the Lamé Hamiltonian in Liealgebraic form. This yields, in a natural way, an explicit formula for both the Lamé polynomials and the classical non-meromorphic Lamé functions in terms of Chebyshev polynomials and of a certain family of weakly orthogonal polynomials.
\end{abstract}

PACS numbers: 03.65.Fd, 02.60.Lj.

\footnotetext{
*Supported in part by DGES Grant PB95-0401.

${ }^{\dagger}$ On leave of absence from Depto. de Física Teórica II, Univ. Complutense de Madrid,
} Spain. 


\section{Introduction}

The Lamé equation,

$$
\psi^{\prime \prime}(x)+\left[E-m \ell(\ell+1) \operatorname{sn}^{2} x\right] \psi(x)=0,
$$

where $\ell$ is a real parameter ${ }^{1}$, and

$$
\operatorname{sn} x \equiv \operatorname{sn}(x \mid m)
$$

is the usual Jacobian elliptic function of modulus $m$, occupies a central position in the theory of differential equations with periodic coefficients. The study of its properties has attracted the attention of many illustrious mathematicians over the last century; classical references are [1-4]. Basic properties of the Lamé equation are as follows. First, it arises by separation of variables in the Laplace equation in ellipsoidal coordinates. Secondly, it possesses two linearly independent $2 K(k)$ or $4 K(k)$-periodic solutions (for characteristic values of $E$ ) if and only if $\ell$ is a nonnegative integer. Here $k=\sqrt{m}$, and $K(k)$ (denoted by $K$ from now on) is the complete elliptic integral of the first kind with parameter $k$ :

$$
K(k)=\int_{0}^{\pi / 2} \frac{d x}{\sqrt{1-k^{2} \sin ^{2} x}} .
$$

Moreover, if $\ell$ is a nonnegative integer there are exactly $\ell$ gaps in the energy spectrum, and the $2 \ell+1$ eigenfunctions associated to the boundaries of the allowed energy bands can be written as homogeneous polynomials of degree $\ell$ in the elliptic functions sn, cn, dn. These polynomial solutions are known as the Lamé polynomials. In the third place, for any $\ell \in \mathbb{R}$ and characteristic values of $E$ the Lamé equation admits two linearly independent solutions of period $8 K$. These solutions are expressible in closed form if and only if $\ell \in \mathbb{N}+1 / 2$, and shall be referred to as the non-meromorphic Lamé functions. For both types of solutions, namely the Lamé polynomials for integer $\ell$ and the non-meromorphic Lamé functions for half-integer $\ell$, the characteristic values of $E$ are the solutions of a certain algebraic equation. We shall use the the term "algebraic" to refer to both types of solutions.

The interest of the Lamé equation is by no means restricted to its mathematical properties. In fact, several important physical applications have been recently proposed in the literature. As first remarked in [5], the Lamé potential can be considered as a realistic model of a one-dimensional crystal. The Lamé equation also describes some class of fluctuations of the sphalerons in

\footnotetext{
${ }^{1}$ Note that without loss of generality we can assume that $\ell \geq-1 / 2$.
} 
the Abelian Higgs model in $1+1$ dimensions, [6]. The quantum fluctuations of the inflation field in certain cosmological models are determined by the Lamé equation, [7,8].

The Lamé equation appears in a natural way in several Lie-algebraic approaches to the Schrödinger equation. Early work in this direction was carried out by Alhassid, Gürsey and Iachello in [5]. In this paper the Lamé equation was obtained by separation of variables in the eigenvalue equation for a Hamiltonian quadratic in the generators of $\mathfrak{s u}(2)$ written in conical coordinates. A different Lie-algebraic representation of the Lamé equation was discovered by Turbiner in [9], where it was shown that for integer $\ell$ the Lamé Hamiltonian belongs to the the enveloping algebra of a Lie algebra of firstorder differential operators with a finite-dimensional module of functions. It follows that a number of eigenvalues and the corresponding eigenfunctions of the Lamé equation can be determined algebraically, i.e., by diagonalizing the finite-dimensional matrix representing the action of the Hamiltonian in the module. This class of Hamiltonians - known in the literature as quasiexactly solvable (QES) - have been the subject of considerable investigation over the last decade; extensive reviews of this field can be found in [10-12]. In particular, it is known that several periodic potentials of physical interest - like the Razavy potential, $[13,14]$, or the so-called associated Lamé potential, [4] - possess the QES property ${ }^{2}$.

In the above mentioned papers the Lamé equation was successfully algebraized only for integer values of $\ell$, corresponding to the Lamé polynomials. A first indirect algebraization of the Lamé equation for half-integer $\ell$ was studied by Ward in [15] in connection with the matrix Nahm equations. The Lamé polynomials and the non-meromorphic Lamé functions appear as solutions of some matrix-valued differential equations related to irreducible representations of $\mathfrak{s o}(3)$. The Lamé potential (and some generalizations thereof) was revisited by Ulyanov and Zaslavskii in [16]. In this paper the Lamé Hamiltonian was obtained from a non-standard realization of $\mathfrak{s u}(2)$ by firstorder differential operators, leading to the algebraic solutions for integer and half-integer values of $\ell$. In [17], Brihaye and Godart introduced yet another indirect algebraization scheme for half-integer values of $\ell$ based on a system of two second-order differential equations.

In this paper we shall present a new direct way to algebraize the Lamé equation which is valid for both the integer and half-integer cases. Our approach is based on the classification of one-dimensional QES potentials by González-López, Kamran, and Olver, [10], and presents some advantages over the similar algebraization considered by Ulyanov and Zaslavskii in [16].

\footnotetext{
${ }^{2} \mathrm{~A}$ notorious exception is the Mathieu potential, which is not QES.
} 
Indeed, the $2 \ell+1$ algebraic points of the spectrum (corresponding to the boundaries of the energy bands for integer $\ell$ and to eigenfunctions of quasimomentum $\pi /(4 K)$ for half-integer $\ell$ ) are the roots of a pair of polynomials of degrees $\ell$ and $\ell+1$ for integer $\ell$, or of a single polynomial of degree $\ell+1 / 2$ for half-integer $\ell$. This compares to the algebraization by Ulyanov and Zaslavskii, for which one needs to find the roots of a polynomial of degree $2 \ell+1$. In the second place, it leads to new general expressions for the algebraic solutions of the Lamé equation in terms of Chebyshev polynomials and of a certain family of weakly-orthogonal polynomials. It should be emphasized that in the algebraization schemes mentioned above explicit solutions are presented only for low (integer or half-integer) values of $\ell$.

The paper is organized as follows. In Section 2, we introduce the basic definitions and show how the Lamé potential can be obtained using the classification of González-López, Kamran, and Olver. The algebraization by Ulyanov and Zaslavskii is briefly discussed in this setting. Section 3 is devoted to the integer case. We find an expansion in terms of Chebyshev polynomials which reveals the structure of the classical Lamé polynomials. This expansion is used to compute the Lamé polynomials for $\ell \leq 4$. In the last section we analyze the half-integer case. We obtain an expansion of the non-meromorphic Lamé functions using again Chebyshev polynomials, and relate our formulae to the classical results of Ince. In particular, we check Ince's formulas for $\ell \leq 3 / 2$, and study in detail the case $\ell=5 / 2$.

\section{Algebraizations of the Lamé equation}

If $n$ is a non-negative integer, it is a standard fact that the differential operators

$$
J_{-}=\partial_{z}, \quad J_{0}=z \partial_{z}-\frac{n}{2}, \quad J_{+}=z^{2} \partial_{z}-n z
$$

are the basis of a representation of the Lie algebra $\mathfrak{s l}(2, \mathbb{R})$ in the space $\mathcal{P}_{n}$ of polynomials of degree at most $n$ in the variable $z,[18,19]$. As a consequence, any differential operator $\mathcal{H}$ which is a polynomial in the operators (2.1) preserves the space $\mathcal{P}_{n}$ (the converse is also true; cf. [20,21]). In particular, $n+1$ eigenfunctions and eigenvalues of $\mathcal{H}$ can be computed algebraically, simply by diagonalizing the finite-dimensional operator obtained by restricting $\mathcal{H}$ to $\mathcal{P}_{n}$. More generally, if we perform the change of variable

$$
z=\zeta(x)
$$


and consider the operator

$$
H(x)=\left.\mu(z) \cdot \mathcal{H} \cdot \frac{1}{\mu(z)}\right|_{z=\zeta(x)},
$$

where $\mu(z)$ is a nowhere vanishing function, then $H$ obviously leaves invariant the $(n+1)$-dimensional vector space

$$
\mu \mathcal{P}_{n}=\left\{\mu(\zeta(x)) p(\zeta(x)) \mid p \in \mathcal{P}_{n}\right\}
$$

Consequently, we can again compute $n+1$ eigenvalues and eigenfunctions of $H$ in an algebraic way, by considering its restriction to the finite-dimensional space $\mu \mathcal{P}_{n}$. We shall say that such an operator $H$ is quasi-exactly solvable (or, for short, QES), [22]. Of particular physical interest is the case in which the operator $\mathcal{H}$ is a polynomial of degree two in the generators (2.1), which we shall write as

$$
\mathcal{H}=-\sum_{a, b=0, \pm} c_{a b} J_{a} J_{b}-\sum_{a=0, \pm} c_{a} J_{a}-c_{*}
$$

Such an operator can be expressed as

$$
-\mathcal{H}=P(z) \partial_{z}^{2}+\left[Q(z)-\frac{1}{2}(n-1) P^{\prime}(z)\right] \partial_{z}+R-\frac{n}{2} Q^{\prime}(z)+\frac{n}{12}(n-1) P^{\prime \prime}(z),
$$

where (see [19])

$$
\begin{aligned}
P(z) & =c_{++} z^{4}+2 c_{+0} z^{3}+\left(2 c_{+-}+c_{00}\right) z^{2}+2 c_{0-} z+c_{--}, \\
Q(z) & =c_{+} z^{2}+c_{0} z+c_{-}, \\
R & =\frac{n}{12}(n+2) c_{00}-\frac{n}{3}(n+2) c_{+-}+c_{*} .
\end{aligned}
$$

Note that, using the Casimir identity

$$
J_{0}^{2}-\frac{1}{2}\left(J_{+} J_{-}+J_{-} J_{+}\right)=\frac{1}{4} n(n+2),
$$

we can take $c_{+-}=0$ without loss of generality. It can be shown, [19], that if $P$ is positive then it is always possible to transform (at least locally) the operator $\mathcal{H}$ into a Schrödinger operator (or Hamiltonian)

$$
H=-\partial_{x}^{2}+V(x)
$$


by an appropriate change of variable and gauge transformation (2.2)(2.3). More precisely, the inverse of the change of variable (2.2) is given by

$$
x=\int_{z_{0}}^{z} \frac{d y}{\sqrt{P(y)}},
$$

while the gauge factor is proportional to

$$
\mu(z)=P(z)^{-n / 4} \exp \left[\int_{z_{0}}^{z} \frac{Q(y)}{2 P(y)} d y\right]
$$

The potential $V(x)$ can be expressed in terms of the coefficients of the gauge Hamiltonian $\mathcal{H}$ as follows

$$
\begin{aligned}
V(x)=-\frac{1}{12 P}[n(n+ & 2)\left(P P^{\prime \prime}-\frac{3}{4} P^{\prime 2}\right) \\
& \left.+3(n+1)\left(Q P^{\prime}-2 P Q^{\prime}\right)-3 Q^{2}\right]\left.\right|_{z=\zeta(x)}-R,
\end{aligned}
$$

where the prime denotes derivative with respect to $z$.

All one-dimensional potentials whose Hamiltonian is QES (in the precise sense explained above) have been completely classified in Ref. [10], modulo a constant translation of the space coordinate $x$. In particular, the Lamé equation (1.1) can be obtained from the third family of periodic potentials (with $\nu=1$ ), given by

$$
V(x)=A \operatorname{sn}^{2} x+B \operatorname{sn} x \operatorname{cn} x+\frac{C \operatorname{sn} x \operatorname{cn} x+D}{\operatorname{dn}^{2} x} .
$$

Note that, for later convenience, the above notation is slightly different from that of Ref. [10]. The potential (2.11) is obtained from (2.10) when

$$
P(z)=\left(1+z^{2}\right)\left[1+(1-m) z^{2}\right] .
$$

Indeed, from (2.8) it follows that, in this case,

$$
z=\frac{\operatorname{sn} x}{\operatorname{cn} x} .
$$

Substituting (2.12) into (2.10) and using (2.13) we find the following explicit 
formula for the coefficients in Eq. (2.11)

$$
\begin{aligned}
A & =\frac{1}{4} m n(n+2)-\frac{c_{0}}{2}(n+1)+\frac{1}{4 m}\left[c_{0}^{2}-\left(c_{+}-c_{-}\right)^{2}\right] \\
B & =\frac{1}{2 m}\left(c_{+}-c_{-}\right)\left[m(n+1)-c_{0}\right] \\
C & =\frac{1}{2 m}\left[c_{+}+(m-1) c_{-}\right]\left[m(n+1)+c_{0}\right] \\
D & =\frac{1}{4}(m-1) n(n+2)+\frac{c_{0}}{2 m}(m-1)(n+1) \\
& \quad+\frac{1}{4 m^{2}}\left[(m-1) c_{0}^{2}+\left(c_{+}+(m-1) c_{-}\right)^{2}\right] .
\end{aligned}
$$

Note that we have taken

$$
\begin{aligned}
c_{*}=\frac{1}{4 m^{2}}\left[(2 m-1) c_{-}^{2}+(1-m)\left(c_{0}^{2}+\right.\right. & \left.\left.2 c_{+} c_{-}\right)-c_{+}^{2}\right] \\
& +\frac{c_{0}}{2 m}(n+1)-\frac{1}{4} n(n+2)
\end{aligned}
$$

in order to eliminate a constant term in the potential. Comparing (1.1) with (2.11) we obtain the system

$$
A=m \ell(\ell+1), \quad B=C=D=0,
$$

which has the following four sets of solutions:

$$
\begin{array}{ll}
n=\ell ; & c_{+}=c_{-}=0, \quad c_{0}=-m \ell \\
n=\ell-1 ; & c_{+}=c_{-}=0, \quad c_{0}=-m(\ell+1) \\
n=\ell-\frac{1}{2} ; & c_{+}=c_{-}=\mathrm{i} \sqrt{1-m}, \quad c_{0}=-m\left(\ell+\frac{1}{2}\right) \\
n=\ell-\frac{1}{2} ; & c_{+}=c_{-}=-\mathrm{i} \sqrt{1-m}, \quad c_{0}=-m\left(\ell+\frac{1}{2}\right) .
\end{array}
$$

The first two solutions are valid when $\ell$ is a non-negative integer $(\ell \geq 1$ for the second solution to exist), while the last two solutions hold when $\ell$ is a positive half-integer. From the previous general discussion it follows that when $\ell$ is a non-negative integer or a positive half-integer a certain number of eigenfunctions and eigenvalues of the Lamé Hamiltonian can be computed in a purely algebraic fashion, by solving the eigenvalue problem of the restriction of the operator $\mathcal{H}$ corresponding to the Hamiltonian (1.1) to the finite-dimensional space $\mathcal{P}_{n}$, where $n$ is given by (2.19)-(2.22). An explicit expression for the gauge Hamiltonian $\mathcal{H}$ can be easily found from 
Eqs. (2.4), (2.6), (2.12), and (2.19)-(2.22), namely

$$
-\mathcal{H}=\left\{\begin{array}{c}
(1-m) J_{+}^{2}+(2-m) J_{0}^{2}+J_{-}^{2}-m \ell J_{0}+c_{*} \\
(1-m) J_{+}^{2}+(2-m) J_{0}^{2}+J_{-}^{2}-m(\ell+1) J_{0}+c_{*} \\
(1-m) J_{+}^{2}+(2-m) J_{0}^{2}+J_{-}^{2}+\mathrm{i} \sqrt{1-m}\left(J_{+}+J_{-}\right) \\
-m\left(\ell+\frac{1}{2}\right) J_{0}+c_{*} \\
(1-m) J_{+}^{2}+(2-m) J_{0}^{2}+J_{-}^{2}-\mathrm{i} \sqrt{1-m}\left(J_{+}+J_{-}\right) \\
-m\left(\ell+\frac{1}{2}\right) J_{0}+c_{*} .
\end{array}\right.
$$

Each of the four cases in this formula corresponds to the respective solution (2.19)-(2.22), and it is understood that in each case the operators $J_{\epsilon}$ $(\epsilon=0,+)$ and the constant $c_{*}$ must be computed using the value of $n$ for the corresponding solution (2.19)-(2.22). Note that Eq. (2.23) is essentially equivalent to Eqs. (7)-(9) of Ref. [9]. The latter equations, however, appear more complicated than (2.23), due to the fact that Ref. [9] uses the Weierstrassian form of Lamé's equation.

Before studying in more detail the four algebraizations (2.19)-(2.22) of the Lamé equation obtained in this section, it is worth pointing out that there are other alternative algebraizations within the general formalism described above. Indeed, from the identity

$$
\operatorname{dn}^{-2}(x+K)=\frac{\operatorname{dn}^{2} x}{1-m}=\frac{1}{1-m}-\frac{m}{1-m} \operatorname{sn}^{2} x,
$$

and the fact that the potentials in Ref. [10] are classified up to constant translations, it can be shown that the Lamé potential is a member of the first two families of periodic QES potentials listed in the latter reference. For the same reason, we could also have derived the Lamé equation by equating to zero the coefficients $A, B$ and $C$ in Eq. (2.11). It can be shown that all these ways of algebraizing the Lamé equation are essentially equivalent to the one adopted in this paper. Finally, there are other less obvious algebraizations of the Lamé equation like, for instance, the non-standard one discussed in Ref. [16], which falls into our framework by taking

$$
\mathcal{H}=(1-m) J_{0}^{2}+\frac{m}{4}\left(J_{+}+J_{-}\right)^{2} .
$$

From Eq. (2.6) it follows that in this case

$P(z)=-\left[\frac{m}{4} z^{4}+\left(1-\frac{m}{2}\right) z^{2}+\frac{m}{4}\right], \quad Q(z)=0, \quad R=(2 m-1) \frac{n(n+2)}{12}$.

It is straightforward to show that the complex change of variable

$$
z=\operatorname{cn} x+\mathrm{i} \operatorname{sn} x
$$


(cf. Eq. (2.8)) and the gauge transformation defined by (2.3) and (2.9) map $\mathcal{H}$ into the Schrödinger operator (2.7) with potential

$$
\begin{aligned}
V(x) & =-m(1-m) \frac{n}{2}\left(\frac{n}{2}+1\right) \frac{\operatorname{sn}^{2} x}{\operatorname{dn}^{2} x} \\
& =m \frac{n}{2}\left(\frac{n}{2}+1\right)\left[\operatorname{sn}^{2}(x+K)-1\right],
\end{aligned}
$$

which is essentially the Lamé potential with $\ell=n / 2$. Since $P(z)$ is negative everywhere and all its roots are complex, it can be mapped to a negative multiple of the polynomial (2.12) by a projective change of variable and gauge transformation. As explained in Ref. [10], this means that the potential (2.24) can be obtained by a suitable choice of parameters in the third family of QES periodic potentials (with pure imaginary frequency $\sqrt{\nu}$ ).

\section{Case $\mathrm{I}: \ell$ is a non-negative integer}

We shall show in this section that when $\ell$ is a non-negative integer the algebraic eigenfunctions of the Lamé Hamiltonian are the classical Lamé polynomials, [4].

From Eqs. (2.9), (2.13), and (2.19)-(2.20) we deduce that in this case the gauge factor verifies

$$
\mu(\zeta(x))= \begin{cases}\operatorname{cn}^{\ell} x, & n=\ell \\ \operatorname{cn}^{\ell-1} x \operatorname{dn} x, & n=\ell-1,\end{cases}
$$

where we have dropped some irrelevant numerical factors. From Eqs. (2.3), (2.13), and (3.1), it follows that when $\ell$ is a non-negative integer then the Lamé Hamiltonian has $\ell+1$ eigenfunctions of the form

$$
\psi(x)=\operatorname{cn}^{\ell} x \cdot \chi\left(\frac{\operatorname{sn} x}{\operatorname{cn} x}\right),
$$

where $\chi$ is a polynomial of degree at most $\ell$. Thus we can write

$$
\psi(x)=\tilde{\chi}(\operatorname{sn} x, \operatorname{cn} x),
$$

where $\tilde{\chi}$ is a homogeneous bivariate polynomial of degree $\ell$. Similarly, if $\ell$ is strictly positive then there are $\ell$ additional eigenfunctions of the Lamé Hamiltonian of the form

$$
\psi(x)=\operatorname{dn} x \cdot \tilde{\eta}(\operatorname{sn} x, \operatorname{cn} x),
$$


where $\tilde{\eta}$ is a homogeneous polynomial in two variables of degree $\ell-1$. Thus, when $\ell$ is a non-negative integer the Lamé Hamiltonian admits $2 \ell+1$ algebraic eigenfunctions of the form (3.2)-(3.3).

To show that the latter $2 \ell+1$ algebraic eigenfunctions are indeed the classical Lamé polynomials, we need to study in more detail Eqs. (3.2)-(3.3). Fortunately, this can be done in a totally systematic way, by applying the general theory developed in Ref. [23] for associating a (weakly) orthogonal polynomial family to a one-dimensional QES Hamiltonian. According to the prescription of the latter reference (cf. [23], Eqs. (30), (33), and (35)), we must choose two different roots $z_{1} \neq z_{2}$ of $P(z)$ such that

$$
(2 j-n-1) P^{\prime}\left(z_{1}\right)+2 Q\left(z_{1}\right) \neq 0, \quad \forall j \geq 1 .
$$

Then the function

$$
\psi_{E}(x)=\mu(\zeta(x))\left(\zeta(x)-z_{2}\right)^{n} \sum_{j=0}^{\infty} \frac{P_{j}(E)}{j !}\left(\frac{\zeta(x)-z_{1}}{\zeta(x)-z_{2}}\right)^{j}
$$

(with $\zeta(x)$ and $\mu(\zeta(x))$ respectively given by Eqs. (2.13) and (3.1)), is a formal solution of the Lamé equation with energy $E$ if and only if the set $\left\{P_{j}(E)\right\}_{j \geq 0}$ satisfies a certain 3 -term recurrence relation. If we normalize $\psi_{E}$ so that $P_{0}=1$, the latter recurrence relation implies that $\left\{P_{j}(E)\right\}_{j \geq 0}$ is a family of (weakly) orthogonal polynomials. Moreover, if the coefficients of the recurrence relation satisfy a positivity condition that we shall state and verify below, the critical polynomial $P_{n+1}$ has $n+1$ different real roots $E_{i}, 0 \leq i \leq n$. Since it is easily shown that each of these roots is also a root of the polynomials $P_{j}(E)$ with $j>n+1$, for $i=0,1, \ldots, n$ the function $\psi_{E_{i}}(x)$ is a genuine solution of the Lamé equation (1.1) with energy $E=E_{i}$. These $n+1$ exact solutions can be obtained algebraically, by computing the $n$ polynomials $P_{1}(E), \ldots, P_{n}(E)$ from the recurrence relation, and in fact coincide with the $n+1$ eigenfunctions of the Lamé Hamiltonian $H$ algebraically computable by diagonalizing the restriction of $H$ to $\mu \mathcal{P}_{n}$ (or, equivalently, the restriction of $\mathcal{H}$ to $\left.\mathcal{P}_{n}\right)$.

It is immediate to verify that (3.4) is satisfied for both solutions (2.19) and (2.20) simply by taking $z_{1}=-\mathrm{i}$. If we choose $z_{2}=\mathrm{i}$, from Eq. (3.5) we obtain the following expression (up to a constant factor) for the $\ell+1$ exact eigenfunctions of the Lamé Hamiltonian coming from the first algebraization $(2.19)$

$$
\psi_{E_{i}}(x)=\sum_{j=0}^{\ell} \frac{(-1)^{j}}{j !} P_{j}\left(E_{i}\right)(\operatorname{cn} x+\mathrm{i} \operatorname{sn} x)^{\ell-j}(\operatorname{cn} x-\mathrm{i} \operatorname{sn} x)^{j}, \quad 0 \leq i \leq \ell,
$$


where the $\ell+1$ energies $E_{i}$ satisfy

$$
P_{\ell+1}\left(E_{i}\right)=0, \quad 0 \leq i \leq \ell
$$

In the same way, the second algebraization (2.20) yields the $\ell$ eigenfunctions

$$
\begin{array}{r}
\psi_{\tilde{E}_{i}}(x)=\operatorname{dn} x \sum_{j=0}^{\ell-1} \frac{(-1)^{j}}{j !} \tilde{P}_{j}\left(\tilde{E}_{i}\right)(\operatorname{cn} x+\mathrm{i} \operatorname{sn} x)^{\ell-1-j}(\operatorname{cn} x-\mathrm{i} \operatorname{sn} x)^{j} \\
0 \leq i \leq \ell-1,
\end{array}
$$

where $\left\{\tilde{P}_{j}\right\}_{j \geq 0}$ is the orthogonal polynomial family constructed from the solution $(2.20)$, and the $\ell$ energies $\tilde{E}_{i}$ are given by

$$
\tilde{P}_{\ell}\left(\tilde{E}_{i}\right)=0, \quad 0 \leq i \leq \ell-1 .
$$

We shall next derive the recurrence relation defining the polynomials $P_{j}$ and $\tilde{P}_{j}$. According to the general procedure described in Ref. [23], the latter relation is determined by the coefficients of the auxiliary polynomials $\hat{P}(w)$, $\hat{Q}(w)$ obtained by applying to $P(z)$ and $Q(z)$ the projective transformation

$$
w=\frac{z-z_{1}}{z-z_{2}}
$$

namely

$$
\begin{aligned}
& \hat{P}(w)=\frac{(1-w)^{4}}{\left(z_{1}-z_{2}\right)^{2}} P\left(\frac{z_{1}-z_{2} w}{1-w}\right), \\
& \hat{Q}(w)=\frac{(1-w)^{2}}{z_{1}-z_{2}} Q\left(\frac{z_{1}-z_{2} w}{1-w}\right)
\end{aligned}
$$

(with $z_{1}=-\mathrm{i}=-z_{2}$ in the present case). From Eqs. (2.12) and (3.11) we easily obtain the following formula for $\hat{P}$, valid for both algebraizations (2.19) and (2.20)

$$
\hat{P}(w)=m w^{3}-2(2-m) w^{2}+m w .
$$

Similarly, Eqs. (2.6) and (3.12) imply that the polynomial $\hat{Q}$ associated to the first algebraization (2.19) is given by

$$
\hat{Q}=\frac{1}{2} m \ell\left(w^{2}-1\right)
$$


while for the second algebraization (2.20) we obtain

$$
\hat{Q}=\frac{1}{2} m(\ell+1)\left(w^{2}-1\right) .
$$

Following Ref. [23], we define new polynomials $\hat{P}_{j}(E)$ and $\hat{\tilde{P}}_{j}(E)$ by

$$
\left.\begin{array}{c}
\hat{P}_{j}(E) \\
\hat{\tilde{P}}_{j}(E)
\end{array}\right\}=\left(\frac{m}{2}\right)^{j} \frac{(2 \ell-1) ! !}{(2 \ell-2 j-1) ! !}\left\{\begin{array}{c}
P_{j}(E) \\
\tilde{P}_{j}(E) .
\end{array}\right.
$$

It is shown in Ref. [23] that the polynomials $\hat{P}_{j}$ satisfy the recurrence relation

$$
\hat{P}_{j+1}=\left(E-b_{j}\right) \hat{P}_{j}-a_{j} \hat{P}_{j-1}, \quad j \geq 0,
$$

with $\hat{P}_{-1}=0$ and

$$
\begin{aligned}
a_{j} & =\frac{1}{4} m^{2} j(2 j-1)(2 \ell-2 j+1)(\ell-j+1), \\
b_{j} & =\frac{1}{2} m \ell(\ell+1)+\frac{1}{2}(2-m)(\ell-2 j)^{2} .
\end{aligned}
$$

Likewise, the polynomials $\hat{\tilde{P}}_{j}$ verify the recurrence relation

$$
\hat{\tilde{P}}_{j+1}=\left(E-\tilde{b}_{j}\right) \hat{\tilde{P}}_{j}-\tilde{a}_{j} \hat{\tilde{P}}_{j-1}, \quad j \geq 0
$$

where $\hat{\tilde{P}}_{-1}=0$, and the coefficients $\tilde{a}_{j}$ and $\tilde{b}_{j}$ are given by

$$
\begin{aligned}
& \tilde{a}_{j}=\frac{1}{4} m^{2} j(2 j+1)(2 \ell-2 j+1)(\ell-j), \\
& \tilde{b}_{j}=\frac{1}{2} m \ell(\ell+1)+\frac{1}{2}(2-m)(\ell-2 j-1)^{2} .
\end{aligned}
$$

Note that from the normalization $P_{0}=\tilde{P}_{0}=1$ and Eq. (3.16) it follows that

$$
\hat{P}_{0}=\hat{\tilde{P}}_{0}=1 .
$$

Furthermore, Eqs. (3.18) and (3.21) imply that the coefficients $a_{j}$ and $\tilde{a}_{j}$ are strictly positive for $1 \leq j \leq \ell$ and $1 \leq j \leq \ell-1$, respectively. By Lemma 1 (Sec. 1.8) of Ref. [4], this positivity condition guarantees that all the roots of the critical polynomials $\hat{P}_{\ell+1}$ and $\hat{\tilde{P}}_{\ell}$ are real and simple, as we had anticipated.

Our next task is to simplify Eqs. (3.6) and (3.8) using the properties of the polynomials $P_{j}$ and $\tilde{P}_{j}$. We shall start by proving the following useful result: 
Proposition 3.1. The algebraic eigenfunctions (3.6) and (3.8) are always either real or purely imaginary.

Proof. Let us rewrite Eqs. (3.6) and (3.8) in terms of the elliptic amplitude $\varphi(x)=\operatorname{am} x$, defined by

$$
\operatorname{cn} x+\mathrm{i} \operatorname{sn} x=e^{\mathrm{i} \varphi(x)} .
$$

We thus obtain the expressions

$$
\psi_{E_{i}}(x)=\sum_{j=0}^{\ell} p_{i j} \mathrm{e}^{\mathrm{i}(\ell-2 j) \varphi(x)}, \quad 0 \leq i \leq \ell,
$$

and

$$
\psi_{\tilde{E}_{r}}(x)=\operatorname{dn} x \sum_{s=0}^{\ell-1} \tilde{p}_{r s} \mathrm{e}^{\mathrm{i}(\ell-2 s-1) \varphi(x)}, \quad 0 \leq r \leq \ell-1,
$$

where we have set, for convenience,

$$
p_{i j}=\frac{(-1)^{j}}{j !} P_{j}\left(E_{i}\right), \quad \tilde{p}_{r s}=\frac{(-1)^{s}}{s !} \tilde{P}_{s}\left(E_{r}\right)
$$

Let us concentrate, for definiteness, on the eigenfunctions of type (3.24). We rewrite Eq. (3.24) in the form

$$
\psi_{E_{i}}(x)=\sum_{0 \leq j<\ell / 2}\left[p_{i j} \mathrm{e}^{\mathrm{i}(\ell-2 j) \varphi(x)}+p_{i, \ell-j} \mathrm{e}^{-\mathrm{i}(\ell-2 j) \varphi(x)}\right]+p_{i, \frac{\ell}{2}},
$$

where we take $p_{i, \ell / 2}=0$ when $\ell$ is odd. Taking into account that, by the recurrence relation (3.17)-(3.19), all the coefficients $p_{i j}$ are real, we conclude from the Eq. (3.27) that

$$
\begin{aligned}
& \operatorname{Re} \psi_{E_{i}}(x)=\sum_{0 \leq j<\ell / 2}\left(p_{i j}+p_{i, \ell-j}\right) \cos (\ell-2 j) \varphi(x)+p_{i, \frac{\ell}{2}}, \\
& \operatorname{Im} \psi_{E_{i}}(x)=\sum_{0 \leq j<\ell / 2}\left(p_{i j}-p_{i, \ell-j}\right) \sin (\ell-2 j) \varphi(x) .
\end{aligned}
$$

Let us now introduce the polynomial family

$$
\pi_{j}(E)=\frac{(-1)^{j}}{j !} P_{j}(E)
$$


in terms of which the coefficients $p_{i j}$ are simply expressed by

$$
p_{i j}=\pi_{j}\left(E_{i}\right), \quad 0 \leq i, j \leq \ell .
$$

Defining two additional families of univariate polynomials $\sigma_{j}(E)$ and $\rho_{j}(E)$ by

$$
\sigma_{j}(E)=\pi_{j}(E)+\pi_{\ell-j}(E), \quad \rho_{j}(E)=\pi_{j}(E)-\pi_{\ell-j}(E),
$$

from Eqs. (3.28)-(3.29) we have

$$
\begin{aligned}
& \operatorname{Re} \psi_{E_{i}} \equiv 0 \quad \Longleftrightarrow \quad \sigma_{j}\left(E_{i}\right)=0 \quad \text { for } \quad 0 \leq j \leq[\ell / 2], \\
& \operatorname{Im} \psi_{E_{i}} \equiv 0 \quad \Longleftrightarrow \quad \rho_{j}\left(E_{i}\right)=0 \quad \text { for } \quad 0 \leq j<\ell / 2
\end{aligned}
$$

(where $[\cdot]$ denotes the integer part). From Eqs. (3.16)-(3.19) and (3.30), it immediately follows that the polynomials $\pi_{j}(E)$ satisfy the three-term recurrence relation

$$
(j+1)(2 \ell-2 j-1) \pi_{j+1}=\frac{2}{m}\left(b_{j}-E\right) \pi_{j}-(2 j-1)(\ell-j+1) \pi_{j-1}
$$

with the initial conditions

$$
\pi_{-1}(E)=0, \quad \pi_{0}(E)=1
$$

where the coefficients $b_{j}$ are defined by Eq. (3.19). It is easy to see that Eq. (3.31) implies that the polynomial families $\left\{\sigma_{j}(E)\right\}_{j \geq 0}$ and $\left\{\rho_{j}(E)\right\}_{j \geq 0}$ satisfy the same three-term recurrence relation (3.32) as the family $\left\{\pi_{j}(E)\right\}_{j \geq 0}$. Moreover, from Eq. (3.7) we obtain

$$
\sigma_{-1}\left(E_{i}\right)=\rho_{-1}\left(E_{i}\right)=0, \quad 0 \leq i \leq \ell .
$$

From this, and the fact that the coefficients $\left\{\sigma_{j}\left(E_{i}\right)\right\}_{j \geq 0}$ and $\left\{\rho_{j}\left(E_{i}\right)\right\}_{j \geq 0}$ satisfy a three-term recursion relation of the type (3.32), we conclude that the vanishing of $\sigma_{0}\left(E_{i}\right)$ or of $\rho_{0}\left(E_{i}\right)$ automatically implies the vanishing of $\sigma_{j}\left(E_{i}\right)$ or of $\rho_{j}\left(E_{i}\right)$, respectively, for all values of $j>0$. Thus we can write

$$
\operatorname{Re} \psi_{E_{i}} \equiv 0 \Longleftrightarrow \sigma_{0}\left(E_{i}\right)=0, \quad \operatorname{Im} \psi_{E_{i}} \equiv 0 \Longleftrightarrow \rho_{0}\left(E_{i}\right)=0 .
$$

To complete the proof of the Proposition, we simply note that Eqs. (3.33)(3.34) and the fact that $\left\{\sigma_{j}\left(E_{i}\right)\right\}_{j \geq 0}$ and $\left\{\rho_{j}\left(E_{i}\right)\right\}_{j \geq 0}$ satisfy the same threeterm recursion relation as $\left\{\pi_{j}(E)\right\}_{j \geq 0}$ imply that

$$
\sigma_{j}\left(E_{i}\right)=\sigma_{0}\left(E_{i}\right) \pi_{j}\left(E_{i}\right), \quad j \geq 0
$$


and, in particular

$$
\begin{aligned}
\sigma_{0}\left(E_{i}\right)=\sigma_{\ell}\left(E_{i}\right)=\sigma_{0} & \left(E_{i}\right) \pi_{\ell}\left(E_{i}\right) \\
& \Longleftrightarrow \sigma_{0}\left(E_{i}\right)\left[1-\pi_{\ell}\left(E_{i}\right)\right] \equiv \sigma_{0}\left(E_{i}\right) \rho_{0}\left(E_{i}\right)=0 .
\end{aligned}
$$

The proof for the eigenfunctions of type (3.25) is totally analogous, and will therefore be omitted.

Let us order, from now on, the $\ell+1$ algebraic eigenvalues $E_{i}$ of the Lamé equation in increasing order, i.e.,

$$
E_{0}<E_{1}<\cdots<E_{\ell-1}<E_{\ell},
$$

and similarly for the $\ell$ eigenvalues $\tilde{E}_{i}$. From Lemma 1 (Sec. 1.8) of Ref. [4] and the positivity of $a_{j}$ for $1 \leq j \leq \ell$ we easily deduce that

$$
\operatorname{sign}\left[(-1)^{\ell} P_{\ell}\left(E_{i}\right)\right]=(-1)^{i},
$$

which implies (by Eq. (3.30)) that $\pi_{\ell}\left(E_{i}\right)$ has the sign of $(-1)^{i}$. Thus for even $i$ we have $\sigma_{0}\left(E_{i}\right)=1+\pi_{\ell}\left(E_{i}\right)>0$, and from (3.36) we conclude that $\rho_{0}\left(E_{i}\right)=0$. In the same way we establish that $\sigma_{0}\left(E_{i}\right)=0$ for odd $i$. From Eq. (3.35) we obtain

$$
\operatorname{Re} \psi_{E_{2 r+1}}=\operatorname{Im} \psi_{E_{2 r}}=0 \text {. }
$$

We can therefore write the $\ell+1$ exact eigenfunctions of type (3.6) of the Lamé equation as the Jacobi-Fourier series

$$
\begin{aligned}
\phi_{2 r}(x) & \equiv \psi_{E_{2 r}}(x)=\sum_{0 \leq j<\ell / 2} \sigma_{2 r, j} \cos (\ell-2 j) \varphi(x)+p_{2 r, \frac{\ell}{2}} \\
\phi_{2 s+1}(x) & \equiv \psi_{E_{2 s+1}}(x)=\sum_{0 \leq j<\ell / 2} \rho_{2 s+1, j} \sin (\ell-2 j) \varphi(x),
\end{aligned}
$$

with

$$
\sigma_{2 r, j} \equiv \sigma_{j}\left(E_{2 r}\right)=p_{2 r, j}+p_{2 r, \ell-j}, \quad \rho_{2 s+1, j} \equiv \rho_{j}\left(E_{2 s+1}\right)=p_{2 s+1, j}-p_{2 s+1, \ell-j},
$$

and

$$
0 \leq r \leq\left[\frac{\ell}{2}\right], \quad 0 \leq s \leq\left[\frac{\ell-1}{2}\right]
$$


We can derive in a totally analogous way an equivalent formula for the $\ell$ eigenfunctions of type (3.27), namely

$$
\begin{gathered}
\tilde{\phi}_{2 r}(x) \equiv \psi_{\tilde{E}_{2 r}}(x)=\operatorname{dn} x\left[\tilde{p}_{2 r, \frac{1}{2}(\ell-1)}+\sum_{0 \leq j<\frac{1}{2}(\ell-1)} \tilde{\sigma}_{2 r, j} \cos (\ell-2 j-1) \varphi(x)\right], \\
\tilde{\phi}_{2 s+1}(x) \equiv \psi_{\tilde{E}_{2 s+1}}(x)=\operatorname{dn} x \sum_{0 \leq j<\frac{1}{2}(\ell-1)} \tilde{\rho}_{2 s+1, j} \sin (\ell-2 j-1) \varphi(x),
\end{gathered}
$$

where now

$$
\tilde{\sigma}_{2 r, j}=\tilde{p}_{2 r, j}+\tilde{p}_{2 r, \ell-j-1}, \quad \tilde{\rho}_{2 s+1, j}=\tilde{p}_{2 s+1, j}-\tilde{p}_{2 s+1, \ell-j-1},
$$

and

$$
0 \leq r \leq\left[\frac{\ell-1}{2}\right], \quad 0 \leq s \leq\left[\frac{\ell}{2}\right]-1 .
$$

Our next step is to further simplify Eqs. (3.39)-(3.46) for the algebraic eigenfunctions of the Lamé Hamiltonian. To this end, recall that by construction the Chebyshev polynomials $T_{j}$ and $U_{j}(j=0,1,2, \ldots)$ satisfy the identities

$$
\begin{aligned}
\cos (j \alpha) & =T_{j}(\cos \alpha), \\
\sin (j \alpha) & =\sin \alpha U_{j-1}(\cos \alpha),
\end{aligned}
$$

where the latter equality is formally valid for $j=0$ if we set $U_{-1}=0$. It follows that

$$
\begin{aligned}
\phi_{2 r}(x) & =\sum_{0 \leq j<\ell / 2} \sigma_{2 r, j} T_{\ell-2 j}(\operatorname{cn} x)+p_{2 r, \frac{\ell}{2}} \\
\phi_{2 s+1}(x) & =\operatorname{sn} x \sum_{0 \leq j<\ell / 2} \rho_{2 s+1, j} U_{\ell-2 j-1}(\operatorname{cn} x),
\end{aligned}
$$

and, similarly,

$$
\begin{aligned}
\tilde{\phi}_{2 r}(x) & =\operatorname{dn} x\left[\tilde{p}_{2 r, \frac{1}{2}(\ell-1)}+\sum_{0 \leq j<\frac{1}{2}(\ell-1)} \tilde{\sigma}_{2 r, j} T_{\ell-2 j-1}(\mathrm{cn} x)\right] \\
\tilde{\phi}_{2 s+1}(x) & =\operatorname{sn} x \operatorname{dn} x \sum_{0 \leq j<\frac{1}{2}(\ell-1)} \tilde{\rho}_{2 s+1, j} U_{\ell-2 j-2}(\operatorname{cn} x) .
\end{aligned}
$$


It is now straightforward to check that Eqs. (3.49)-(3.52) encompass the eight types of classical Lamé polynomials listed in Ref. [4]. Indeed, note first of all that the polynomials $T_{j}(t)$ and $U_{j}(t)$ have the parity of $(-1)^{j}$ under the reflection $x \mapsto-x$. Therefore the formulas

$$
\tilde{T}_{2 j}(t)=\frac{T_{2 j+1}(t)}{t}, \quad \tilde{U}_{2 j}(t)=\frac{U_{2 j+1}(t)}{t} ; \quad j=0,1,2, \ldots
$$

define even polynomials $\tilde{T}_{2 j}(t)$ and $\tilde{U}_{2 j}(t)$ of degree $2 j$. If $\ell=2 N(N=$ $1,2, \ldots)$, Eqs. (3.49)-(3.52) yield the following four types of polynomial in sn, cn, dn - solutions of the Lamé equation:

$$
\begin{aligned}
\phi_{2 r}(x) & =p_{2 r, N}+\sum_{j=0}^{N-1} \sigma_{2 r, j} T_{2 N-2 j}(\operatorname{cn} x) \equiv \alpha_{r}\left(\operatorname{sn}^{2} x\right) \\
\phi_{2 s+1}(x) & =\operatorname{sn} x \operatorname{cn} x \sum_{j=0}^{N-1} \rho_{2 s+1, j} \tilde{U}_{2 N-2 j-2}(\operatorname{cn} x) \equiv \operatorname{sn} x \operatorname{cn} x \beta_{s}\left(\operatorname{sn}^{2} x\right) \\
\tilde{\phi}_{2 s}(x) & =\operatorname{cn} x \operatorname{dn} x \sum_{j=0}^{N-1} \tilde{\sigma}_{2 s, j} \tilde{T}_{2 N-2 j-2}(\operatorname{cn} x) \equiv \operatorname{cn} x \operatorname{dn} x \tilde{\alpha}_{s}\left(\operatorname{sn}^{2} x\right) \\
\tilde{\phi}_{2 s+1}(x) & =\operatorname{sn} x \operatorname{dn} x \sum_{j=0}^{N-1} \tilde{\rho}_{2 s+1, j} \tilde{U}_{2 N-2 j-2}(\operatorname{cn} x) \equiv \operatorname{sn} x \operatorname{dn} x \tilde{\beta}_{s}\left(\operatorname{sn}^{2} x\right),
\end{aligned}
$$

where

$$
0 \leq r \leq N, \quad 0 \leq s \leq N-1
$$

and $\alpha_{r}(t), \beta_{s}(t), \tilde{\alpha}_{s}(t)$, and $\tilde{\beta}_{s}(t)$ are polynomials of respective degrees

$$
\operatorname{deg} \alpha_{r}=N, \quad \operatorname{deg} \beta_{s}=\operatorname{deg} \tilde{\alpha}_{s}=\operatorname{deg} \tilde{\beta}_{s}=N-1 .
$$

Similarly, if $\ell=2 N+1(N=0,1,2, \ldots)$ the exact solutions of the Lamé 
equation (3.49)-(3.52) can be written as follows:

$$
\begin{aligned}
\phi_{2 r}(x) & =\operatorname{cn} x \sum_{j=0}^{N} \sigma_{2 r, j} \tilde{T}_{2 N-2 j}(\operatorname{cn} x) \equiv \operatorname{cn} x \gamma_{r}\left(\operatorname{sn}^{2} x\right) \\
\phi_{2 r+1}(x) & =\operatorname{sn} x \sum_{j=0}^{N} \rho_{2 r+1, j} U_{2 N-2 j}(\operatorname{cn} x) \equiv \operatorname{sn} x \delta_{r}\left(\operatorname{sn}^{2} x\right) \\
\tilde{\phi}_{2 r}(x) & =\operatorname{dn} x\left[\tilde{p}_{2 r, N}+\sum_{j=0}^{N-1} \tilde{\sigma}_{2 r, j} T_{2 N-2 j}(\operatorname{cn} x)\right] \equiv \operatorname{dn} x \tilde{\gamma}_{r}\left(\operatorname{sn}^{2} x\right) \\
\tilde{\phi}_{2 s+1}(x) & =\operatorname{sn} x \operatorname{cn} x \operatorname{dn} x \sum_{j=0}^{N-1} \tilde{\rho}_{2 s+1, j} \tilde{U}_{2 N-2 j-2}(\operatorname{cn} x) \equiv \operatorname{sn} x \operatorname{cn} x \operatorname{dn} x \tilde{\delta}_{s}\left(\operatorname{sn}^{2} x\right)
\end{aligned}
$$

where the range of the indices $r, s$ is still given by Eq. (3.58), and $\gamma_{r}(t), \delta_{r}(t)$, $\tilde{\gamma}_{r}(t)$, and $\tilde{\delta}_{s}(t)$ are polynomials with

$$
\operatorname{deg} \gamma_{r}=\operatorname{deg} \delta_{r}=\operatorname{deg} \tilde{\gamma}_{r}=N, \quad \operatorname{deg} \tilde{\delta}_{s}=N-1 .
$$

\subsection{Examples}

The explicit expressions for the Lamé polynomials in terms of Chebyshev polynomials derived at the end of the previous section can be used to algorithmically compute the Lamé polynomials and their corresponding energies for low values of $\ell$.

When $\ell=1$, Eqs. (3.60)-(3.63) for $N=0$ immediately yield the following well-known formulas for the Lamé polynomials of order 1 :

$$
\phi_{0}(x) \propto \operatorname{cn} x, \quad \phi_{1}(x) \propto \operatorname{sn} x, \quad \tilde{\phi}_{0} \propto \operatorname{dn} x .
$$

The critical polynomials $\hat{P}_{2}$ and $\hat{\tilde{P}}_{1}$ are easily computed using the recurrence relations (3.17)-(3.22), with the result

$$
\hat{P}_{2}(E)=E^{2}-(m+2) E+m+1, \quad \hat{\tilde{P}}_{1}(E)=E-m .
$$

The energies associated to the eigenfunctions (3.65) are just the roots of each of the latter polynomials ordered increasingly, namely

$$
E_{0}=1, \quad E_{1}=m+1 ; \quad \tilde{E}_{0}=m .
$$


For $\ell=2$ the critical polynomials are

$$
\begin{aligned}
& \hat{P}_{3}(E)=E^{3}-(5 m+8) E^{2}+4\left(m^{2}+8 m+4\right) E-12 m(m+4), \\
& \hat{\tilde{P}}_{2}(E)=E^{2}-(5 m+2) E+(m+1)(4 m+1) .
\end{aligned}
$$

The energies corresponding to the Lamé polynomials of order two are therefore given by

$$
\begin{aligned}
& \left.\begin{array}{l}
E_{0} \\
E_{2}
\end{array}\right\}=2\left(1+m \mp \sqrt{m^{2}-m+1}\right), \quad E_{1}=4+m ; \\
& \tilde{E}_{0}=m+1, \quad \tilde{E}_{1}=4 m+1 .
\end{aligned}
$$

Note that the factor of 2 in (3.70) is missing in Ref. [4], p. 205. The Lamé polynomials are easily computed using (3.54)-(3.57) with $N=1$, with the following result:

$$
\begin{aligned}
& \left.\begin{array}{c}
-\frac{\phi_{0}}{4} \\
-\frac{\phi_{2}}{4}
\end{array}\right\}=\operatorname{sn}^{2} x-\frac{1}{3 m}\left(1+m \pm \sqrt{m^{2}-m+1}\right), \\
& \frac{\phi_{1}}{4}=\operatorname{sn} x \operatorname{cn} x ; \\
& \frac{\tilde{\phi}_{0}}{2}=\operatorname{cn} x \operatorname{dn} x, \quad \frac{\tilde{\phi}_{1}}{4}=\operatorname{sn} x \operatorname{dn} x .
\end{aligned}
$$

When $\ell=3$ the critical polynomials are given by

$$
\begin{aligned}
& \hat{P}_{4}(E)=\left[E^{2}-2(5+2 m) E+3(3+8 m)\right] \\
& \times\left[E^{2}-10(1+m) E+3\left(3 m^{2}+26 m+3\right)\right], \\
& \hat{\tilde{P}}_{3}(E)=[E-4(m+1)]\left[E^{2}-2(2+5 m) E+3 m(3 m+8],\right.
\end{aligned}
$$

from which the following exact energies are computed:

$$
\begin{aligned}
& \left.\begin{array}{l}
E_{0} \\
E_{2}
\end{array}\right\}=2 m+5 \mp 2 \sqrt{m^{2}-m+4}, \\
& \left.\begin{array}{l}
E_{1} \\
E_{3}
\end{array}\right\}=5(m+1) \mp 2 \sqrt{4 m^{2}-7 m+4} \text {; } \\
& \left.\begin{array}{c}
\tilde{E}_{0} \\
\tilde{E}_{2}
\end{array}\right\}=5 m+2 \mp 2 \sqrt{4 m^{2}-m+1} \text {, } \\
& \tilde{E}_{1}=4(m+1) \text {. }
\end{aligned}
$$


From Eqs. (3.60)-(3.63) with $N=1$, the corresponding Lamé polynomials are given by

$$
\begin{aligned}
& \left.\begin{array}{c}
-\frac{\phi_{0}}{8} \\
-\frac{\phi_{2}}{8}
\end{array}\right\}=\operatorname{cn} x\left[\operatorname{sn}^{2} x-\frac{1}{5 m}\left(m+2 \pm \sqrt{m^{2}-m+4}\right)\right], \\
& \left.\begin{array}{c}
-\frac{\phi_{1}}{8} \\
-\frac{\phi_{3}}{8}
\end{array}\right\}=\operatorname{sn} x\left[\operatorname{sn}^{2} x-\frac{1}{5 m}\left(2(m+1) \pm \sqrt{4 m^{2}-7 m+4}\right)\right] \text {; } \\
& \left.\begin{array}{r}
-\frac{\tilde{\phi}_{0}}{4} \\
-\frac{\tilde{\phi}_{2}}{4}
\end{array}\right\}=\operatorname{dn} x\left[\operatorname{sn}^{2} x-\frac{1}{5 m}\left(2 m+1 \pm \sqrt{4 m^{2}-m+1}\right)\right], \\
& \frac{\tilde{\phi}_{1}}{4}=\operatorname{sn} x \operatorname{cn} x \operatorname{dn} x .
\end{aligned}
$$

The latter formulas for the Lamé polynomials of order three and their corresponding energies are seen to coincide with those given in Ref. [4].

When $\ell$ is greater than three, it becomes increasingly more difficult to compute the Lamé polynomials of order $\ell$ and their corresponding eigenfunctions in closed form. This is essentially due to the fact that the high degree of the critical polynomials $\hat{P}_{\ell}$ and $\hat{\tilde{P}}_{\ell+1}$ in general makes it impossible to exactly compute the energies $E_{i}$ and $\tilde{E}_{i}$, which enter in the definition of the coefficients $p_{i j}$ and $\tilde{p}_{i j}$. For example, for $\ell=4$ the critical polynomials $\hat{P}_{5}$ and $\hat{\tilde{P}}_{4}$ are given by

$$
\begin{aligned}
\hat{P}_{5}(E)= & {\left[E^{2}-10 E(2+m)+64+136 m+9 m^{2}\right] } \\
\times & {\left[E^{3}-20 E^{2}(1+m)+16 E\left(4+21 m+4 m^{2}\right)-640 m(1+m)\right] } \\
\hat{\tilde{P}}_{4}(E)= & {\left[E^{2}-10 E(1+m)+9+46 m+9 m^{2}\right] } \\
& \quad \times\left[E^{2}-10 E(1+2 m)+9+136 m+64 m^{2}\right] .
\end{aligned}
$$

It is not difficult to verify that the roots of the quadratic factor of $P_{5}$ are the energies $E_{1}$ and $E_{3}$, so that we have the exact formula

$$
\left.\begin{array}{l}
E_{1} \\
E_{3}
\end{array}\right\}=5(m+2) \mp \sqrt{4 m^{2}-9 m+9} .
$$


The corresponding Lamé polynomials are easily computed using Eq. (3.55):

$$
\left.\begin{array}{r}
-\frac{\phi_{1}}{16} \\
-\frac{\phi_{3}}{16}
\end{array}\right\}=\operatorname{sn} x \operatorname{cn} x\left[\operatorname{sn}^{2} x-\frac{1}{7 m}\left(2 m+3 \pm \sqrt{4 m^{2}-9 m+9}\right)\right] \text {. }
$$

Similarly, the fact that $\tilde{P}_{4}$ factorizes into two quadratic polynomials allows us to exactly compute the energies $\tilde{E}_{i}$ in this case, obtaining

$$
\begin{aligned}
& \left.\begin{array}{c}
\tilde{E}_{0} \\
\tilde{E}_{2}
\end{array}\right\}=5(m+1) \mp 2 \sqrt{4 m^{2}+m+4}, \\
& \left.\begin{array}{c}
\tilde{E}_{1} \\
\tilde{E}_{3}
\end{array}\right\}=5(2 m+1) \mp 2 \sqrt{9 m^{2}-9 m+4} \text {. }
\end{aligned}
$$

By Eqs. (3.56)-(3.57), the corresponding eigenfunctions are given by

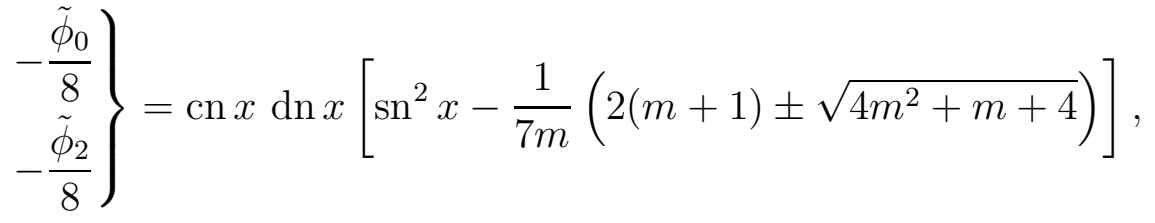

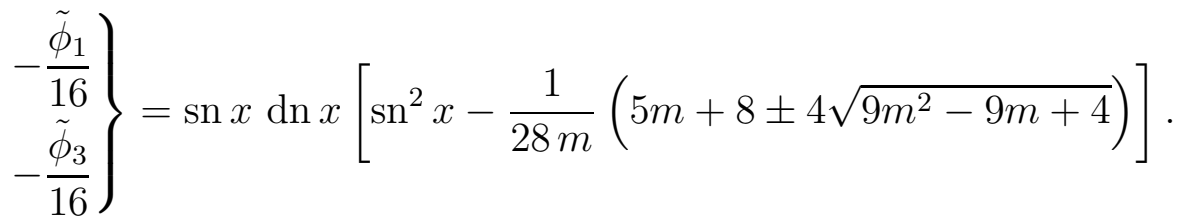

The remaining Lamé polynomials of order four can be expressed using Eq. (3.54) in terms of the three roots $E_{i}(i=0,2,4)$ of the cubic factor of $\hat{P}_{5}$, with the following result:

$$
\begin{aligned}
\frac{\phi_{i}}{16}=\operatorname{sn}^{4} x- & \frac{1}{14 m}\left[16(m+1)-E_{i}\right] \operatorname{sn}^{2} x \\
& +\frac{1}{280 m^{2}}\left[E_{i}^{2}-20(m+1) E_{i}+64 m^{2}+296 m+64\right] .
\end{aligned}
$$

For instance, for $m=1 / 2$ the roots $E_{0}, E_{2}$ and $E_{4}$ can be computed in closed form:

$$
\left.\begin{array}{l}
E_{0} \\
E_{4}
\end{array}\right\}=10 \mp 2 \sqrt{13}, \quad E_{2}=10
$$


Substituting into Eq. (3.93) we obtain the following exact expressions for the corresponding Lamé polynomials for $m=1 / 2$ :

$$
\begin{gathered}
\left.\begin{array}{l}
\frac{\phi_{0}}{16} \\
\frac{\phi_{2}}{16}
\end{array}\right\}=\operatorname{sn}^{4} x-\frac{2}{7}(7 \pm \sqrt{13}) \operatorname{sn}^{2} x+\frac{2}{7}(4 \pm \sqrt{13}) \\
\frac{\phi_{2}}{16}=\operatorname{sn}^{4} x-2 \operatorname{sn}^{2} x+\frac{2}{5}
\end{gathered}
$$

\section{Case II: $\ell$ is a positive half-integer}

This is the most interesting case, since we shall see that it leads to the nonmeromorphic Lamé functions, whose algebraization has proved more difficult than that of the Lamé polynomials.

The gauge factor can be computed from Eqs. (2.9), (2.13), and (2.21)(2.22), with the result

$$
\mu(\zeta(x))=\operatorname{cn}^{n} x\left(\operatorname{cn} x \pm \mathrm{i} k^{\prime} \operatorname{sn} x\right)^{1 / 2} .
$$

The upper and lower signs in this formula correspond, respectively, to the solutions (2.21) and (2.22),

$$
n=\ell-\frac{1}{2}
$$

and we are using the customary notation

$$
k=\sqrt{m}, \quad k^{\prime}=\sqrt{1-m} .
$$

Thus when $\ell$ is a positive half-integer the Lamé equation has $2 n+2=2 \ell+1$ algebraic solutions of the form

$$
\psi^{ \pm}(x)=\operatorname{cn}^{n} x\left(\operatorname{cn} x \pm \mathrm{i} k^{\prime} \operatorname{sn} x\right)^{1 / 2} \chi^{ \pm}\left(\frac{\operatorname{sn} x}{\operatorname{cn} x}\right),
$$

where $\chi^{ \pm}$is a polynomial of degree at most $n$.

We shall now use the same strategy as in the previous section to simplify the eigenfunctions (4.4). It is straightforward to check that in this case only the two roots $\pm \mathrm{i} / k^{\prime}$ of the polynomial $P(z)$ in (2.12) satisfy (3.4). For reasons that shall become clear later, we shall choose

$$
z_{1}=\mp \frac{\mathrm{i}}{k^{\prime}}, \quad z_{2}= \pm \frac{\mathrm{i}}{k^{\prime}}
$$


where again the upper sign corresponds to (2.21) and the lower sign to (2.22). Using the same convention for the eigenfunctions $\psi_{E_{i}^{ \pm}}^{ \pm}$and the polynomials $P_{j}^{ \pm}$, a straightforward calculation yields

$$
\psi_{E_{i}^{ \pm}}^{ \pm}(x)=\sum_{j=0}^{n} \frac{(-1)^{j}}{j !} P_{j}^{ \pm}\left(E_{i}^{ \pm}\right)\left(\operatorname{cn} x \pm \mathrm{i} k^{\prime} \operatorname{sn} x\right)^{n-j+\frac{1}{2}}\left(\operatorname{cn} x \mp \mathrm{i} k^{\prime} \operatorname{sn} x\right)^{j} .
$$

We have dropped an inessential constant factor in the latter formula, and the energies $E_{i}^{ \pm}$are defined by

$$
P_{n+1}^{ \pm}\left(E_{i}^{ \pm}\right)=0, \quad 0 \leq i \leq n .
$$

(We shall prove below that the roots of $P_{n+1}^{ \pm}$are indeed real and simple.)

We now compute the polynomials $P_{j}^{ \pm}$, or more precisely the recurrence relation defining them. In principle, we should use the notation $\hat{P}^{ \pm}(w)$ and $\hat{Q}^{ \pm}(w)$ to denote the polynomials $\hat{P}(w), \hat{Q}(w)$ in Eqs. (3.11)-(3.12) corresponding to each of the two cases (2.21) and (2.22). Remarkably, a direct calculation shows that this is actually unnecessary, since

$$
\begin{aligned}
& \hat{P}^{+}(w)=\hat{P}^{-}(w) \equiv \hat{P}(w)=-\left[m w^{3}+2(2-m) w^{2}+m w\right] . \\
& \hat{Q}^{+}(w)=\hat{Q}^{-}(w) \equiv \hat{Q}(w)=\frac{m}{2}(n+2) w^{2}+(2-m) w-\frac{1}{2} m n .
\end{aligned}
$$

This implies that the recurrence relations determining the two sets of orthogonal polynomials $\left\{P_{j}^{ \pm}(E)\right\}_{j \geq 0}$ are in fact the same ${ }^{3}$. Since we have normalized both sets so that $P_{0}^{ \pm}(E)=1$, it follows that both polynomial families are identical, namely

$$
P_{j}^{+}(E)=P_{j}^{-}(E) \equiv P_{j}(E), \quad j=0,1,2, \ldots
$$

In particular, the above equality for $j=n+1$ and Eq. (4.6) show that

$$
E_{i}^{+}=E_{i}^{-} \equiv E_{i}, \quad 0 \leq i \leq n .
$$

Moreover, to each of these $n+1$ energies correspond two algebraic eigenfunctions of the form (4.5), which on account of (4.9) can be written as

$$
\psi_{E_{i}}^{ \pm}=\sum_{j=0}^{n} \frac{(-1)^{j}}{j !} P_{j}\left(E_{i}\right)\left(\operatorname{cn} x \pm \mathrm{i} k^{\prime} \operatorname{sn} x\right)^{n-j+\frac{1}{2}}\left(\operatorname{cn} x \mp \mathrm{i} k^{\prime} \operatorname{sn} x\right)^{j} .
$$

\footnotetext{
${ }^{3}$ From Eq. (2.18) we have $c_{*}^{+}=c_{*}^{-}$, and from the fact that $R$ is invariant under projective transformations, [19], and Eqs. (2.6) and (4.7) we obtain

$$
\hat{c}_{*}^{+}=\frac{n}{12}(n+2)\left(c_{00}-\hat{c}_{00}^{+}\right)+c_{*}^{+}=\hat{c}_{*}^{-} .
$$
}


From Eqs. (4.7)-(4.8) we see that the coefficients of the polynomials $\hat{P}(w)$ and $\hat{Q}(w)$ are all real. This implies, [23], that the coefficients of the recurrence relation determining the polynomials $P_{j}(E)$ are also real. Since $P_{0}(E)=$ 1 , it follows that $P_{j}(E)$ is a polynomial with real coefficients, for all $j=$ $0,1, \ldots$ From Eq. (4.11) and the positivity of the coefficient $a_{j}$ for $1 \leq j \leq n$ (cf. Eq. (4.14) below), it follows that all the energies $E_{i}(0 \leq i \leq n)$ are real and simple, and therefore

$$
\psi_{E_{i}}^{-}=\overline{\psi_{E_{i}}^{+}}, \quad 0 \leq i \leq n,
$$

where the overbar denotes complex conjugation. If we set

$$
\psi_{i}(x) \equiv \psi_{E_{i}}^{+}(x), \quad 0 \leq i \leq n
$$

then for each root $E_{i}$ of $P_{n+1}$ the Lamé equation has two algebraic real-valued solutions given by

$$
\phi_{i}^{1}(x)=\operatorname{Re} \psi_{i}(x), \quad \phi_{i}^{2}(x)=\operatorname{Im} \psi_{i}(x) .
$$

The recurrence relation satisfied by the polynomials $P_{j}(E)$ can be easily written down from (4.7)-(4.8) using the prescription of Ref. [23]. Indeed, if, following the latter reference, we write

$$
\frac{P_{j}(E)}{j !}=\left(\frac{2}{k}\right)^{2 j} \frac{\hat{P}_{j}(E)}{(2 j) !},
$$

then the polynomials $\hat{P}_{j}$ satisfy a three-term recurrence relation of the form (3.17), with

$$
\begin{aligned}
a_{j} & =\frac{1}{4} m^{2} j(2 j-1)(2 n-2 j+3)(n-j+1), \\
b_{j} & =\frac{1}{4}(2 n+1)(m+2 n+1)-(2-m) j(2 n-2 j+1) .
\end{aligned}
$$

We are ready to show that, when $\ell$ is a half-integer, the $2 \ell+1$ algebraic solutions of the Lamé equation of the form (4.12)-(4.11) obtained in the previous section are precisely the non-meromorphic Lamé functions.

To this end, we need to simplify Eq. (4.11). In the first place, since

$$
\left|\operatorname{cn} x \pm \mathrm{i} k^{\prime} \operatorname{sn} x\right|=\operatorname{dn} x
$$

(recall that $\operatorname{dn} x$ is positive for all real $x$ ), we can write

$$
\operatorname{cn} x \pm \mathrm{i} k^{\prime} \operatorname{sn} x=\operatorname{dn} x \mathrm{e}^{ \pm \mathrm{i} \theta(x)},
$$


so that

$$
\cos \theta(x)=\frac{\operatorname{cn} x}{\operatorname{dn} x}, \quad \sin \theta(x)=k^{\prime} \frac{\operatorname{sn} x}{\operatorname{dn} x} .
$$

Secondly, we have

$$
\left(\operatorname{cn} x+\mathrm{i} k^{\prime} \operatorname{sn} x\right)^{1 / 2}= \pm \frac{1}{\sqrt{2}}(\sqrt{\operatorname{dn} x+\operatorname{cn} x}+\mathrm{i} \epsilon(x) \sqrt{\operatorname{dn} x-\operatorname{cn} x})
$$

where we have set

$$
\epsilon(x)=\operatorname{sign}(\operatorname{sn} x) .
$$

Note that the functions $\sqrt{\operatorname{dn} x+\operatorname{cn} x}$ and $\epsilon(x) \sqrt{\operatorname{dn} x-\operatorname{cn} x}$ are $C^{\infty}$ on the whole real line except at the points $x=2(2 r+1) K$. We shall also find useful in the sequel the identities

$$
\sqrt{\operatorname{dn} x \pm \operatorname{cn} x}=\epsilon(x) \frac{\operatorname{dn} x \pm \operatorname{cn} x}{k^{\prime} \operatorname{sn} x} \sqrt{\operatorname{dn} x \mp \operatorname{cn} x} .
$$

From (4.17) it follows that

$$
\begin{aligned}
\sum_{j=0}^{n} p_{i j}\left(\operatorname{cn} x+\mathrm{i} k^{\prime} \operatorname{sn} x\right)^{n-j}\left(\operatorname{cn} x-\mathrm{i} k^{\prime} \operatorname{sn} x\right)^{j} & \\
& =\operatorname{dn}^{n} x \sum_{j=0}^{n} p_{i j} \mathrm{e}^{\mathrm{i}(n-2 j) \theta(x)},
\end{aligned}
$$

where the coefficients $p_{i j}$ are again defined by Eq. (3.26). The sum in the latter equation is of the same type as (3.24), and therefore can be expressed as follows:

$$
\begin{aligned}
\sum_{j=0}^{n} p_{i j} \mathrm{e}^{\mathrm{i}(n-2 j) \theta(x)}= & p_{i, n / 2}+\sum_{0 \leq j<n / 2} \sigma_{i j} T_{n-2 j}(\cos \theta(x)) \\
& +\mathrm{i} \sin \theta(x) \sum_{0 \leq j<n / 2} \rho_{i j} U_{n-2 j-1}(\cos \theta(x)) \\
= & p_{i, n / 2}+\sum_{0 \leq j<n / 2} \sigma_{i j} T_{n-2 j}(\operatorname{cd} x) \\
& +\mathrm{i} k^{\prime} \operatorname{sd} x \sum_{0 \leq j<n / 2} \rho_{i j} U_{n-2 j-1}(\operatorname{cd} x)
\end{aligned}
$$

where we have used the customary abbreviations

$$
\operatorname{cd} x \equiv \frac{\operatorname{cn} x}{\operatorname{dn} x}, \quad \operatorname{sd} x \equiv \frac{\operatorname{sn} x}{\operatorname{dn} x},
$$


and we have again set

$$
\sigma_{i j}=p_{i j}+p_{i, n-j}, \quad \rho_{i j}=p_{i j}-p_{i, n-j} .
$$

From Eqs. (4.19) and (4.21) (dropping the constant factor $\pm 1 / \sqrt{2}$ ) we easily obtain

$$
\begin{aligned}
\phi_{i}^{1}(x)=\sqrt{\operatorname{dn} x+\operatorname{cn} x} \operatorname{dn}^{n} x & {\left[p_{i, n / 2}+\sum_{0 \leq j<n / 2} \sigma_{i j} T_{n-2 j}(\operatorname{cd} x)\right.} \\
& \left.+(\operatorname{cd} x-1) \sum_{0 \leq j<n / 2} \rho_{i j} U_{n-2 j-1}(\operatorname{cd} x)\right] .
\end{aligned}
$$

An analogous calculation leads to the following expression for the second solution $\phi_{i}^{2}(x)$ :

$$
\begin{aligned}
\phi_{i}^{2}(x)=\epsilon(x) \sqrt{\operatorname{dn} x-\operatorname{cn} x} & \operatorname{dn}^{n} x\left[p_{i, n / 2}+\sum_{0 \leq j<n / 2} \sigma_{i j} T_{n-2 j}(\operatorname{cd} x)\right. \\
& \left.+(\operatorname{cd} x+1) \sum_{0 \leq j<n / 2} \rho_{i j} U_{n-2 j-1}(\operatorname{cd} x)\right] .
\end{aligned}
$$

Let us now study in more detail the properties of the $2 \ell+1$ solutions of the Lamé equation of the form (4.25)-(4.26) that we have just obtained. From the differentiability properties of the functions $\sqrt{\operatorname{dn} x+\operatorname{cn} x}$ and $\epsilon(x) \sqrt{\operatorname{dn} x-\operatorname{cn} x}$ mentioned above, it follows that $\phi_{i}^{1}(x)$ and $\phi_{i}^{2}(x)$ are $C^{\infty}$ solutions of the Lamé equation (1.1) with $E=E_{i}$ in the open interval $(-2 K, 2 K)$, and are clearly continuous on $[-2 K, 2 K]$. The product of $\operatorname{dn}^{n} x$ with the functions within square brackets in Eqs. (4.25) and (4.26) defines two $C^{\infty}$ functions on the whole real line (they are in fact polynomials in $\operatorname{sn} x, \operatorname{cn} x, \operatorname{dn} x$ ), with (real) period $4 K$. On the other hand, it is immediate to check that if we prolong $f(x)=\sqrt{\operatorname{dn} x+\operatorname{cn} x}$ or $f(x)=\epsilon(x) \sqrt{\operatorname{dn} x-\operatorname{cn} x}$ outside the interval $[-2 K, 2 K]$ anti-periodically, i.e. requiring that

$$
f(x+4 K)=-f(x),
$$

then $f$ becomes a $C^{\infty}$ function. (Indeed, in a neighborhood of $\pm 2 K$ the prolongations of $\sqrt{\operatorname{dn} x+\operatorname{cn} x}$ and $\epsilon(x) \sqrt{\operatorname{dn} x-\operatorname{cn} x}$ are equal, respectively, to $\pm k^{\prime} \operatorname{sn} x / \sqrt{\operatorname{dn} x-\operatorname{cn} x}$ and $\pm \sqrt{\operatorname{dn} x-\operatorname{cn} x}$, the latter functions being obviously $C^{\infty}$ at $\pm 2 K$.) It follows that, if we define $\phi_{i}^{1}(x)$ and $\phi_{i}^{2}(x)$ outside 
$[-2 K, 2 K]$ by the prescription (4.27), then $\phi_{i}^{1}$ and $\phi_{i}^{2}$ are $C^{\infty}$ on the whole real line, and have (real) period $8 K$. Furthermore, from Eqs. (4.25) and (4.26) it follows that $\phi_{i}^{1}(x)$ and $\phi_{i}^{2}(x)$ are, respectively, even and odd functions of the variable $x$. It is also clear from the well-known equalities

$$
\operatorname{sn}(x+2 K)=-\operatorname{sn} x, \quad \operatorname{cn}(x+2 K)=-\operatorname{cn} x, \quad \operatorname{dn}(x+2 K)=\operatorname{dn} x,
$$

that $\phi_{i}^{1}$ and $\phi_{i}^{2}$ are related by

$$
\phi_{i}^{2}(x)=\phi_{i}^{1}(x+2 K) .
$$

From this equality and the anti-periodicity condition (4.27) it follows that

$$
\left[\phi_{i}^{1}(x+2 K) \pm \mathrm{i} \phi_{i}^{2}(x+2 K)\right]=\mp \mathrm{i}\left[\phi_{i}^{1}(x) \pm \mathrm{i} \phi_{i}^{2}(x)\right],
$$

so that the quasi-momentum associated to each of the $2 \ell+1$ algebraic energies $E_{i}$ is equal to $\pi /(4 K)$.

When $n$ is odd, it is convenient to rewrite Eq. (4.25) in the following way:

$$
\begin{aligned}
& \phi_{i}^{1}(x)=\sqrt{\operatorname{dn} x+\operatorname{cn} x} \operatorname{dn}^{n} x {\left[\operatorname{cd} x \sum_{j=0}^{\frac{1}{2}(n-1)} \sigma_{i j} \tilde{T}_{n-2 j-1}(\operatorname{cd} x)\right.} \\
&\left.+(\operatorname{cd} x-1) \sum_{j=0}^{\frac{1}{2}(n-1)} \rho_{i j} U_{n-2 j-1}(\operatorname{cd} x)\right] \\
&=\sqrt{\operatorname{dn} x+\operatorname{cn} x} \operatorname{dn}^{n-1} x\left[\operatorname{cn} x \sum_{j=0}^{\frac{1}{2}(n-1)} \sigma_{i j} \tilde{T}_{n-2 j-1}(\operatorname{cd} x)\right. \\
&\left.+(\operatorname{cn} x-\operatorname{dn} x) \sum_{j=0}^{\frac{1}{2}(n-1)} \rho_{i j} U_{n-2 j-1}(\operatorname{cd} x)\right] .
\end{aligned}
$$

Taking into account the parity properties of the Chebyshev polynomials, and the fact that $\operatorname{cn}^{2} x$ and $\operatorname{dn}^{2} x$ can be expressed linearly in terms of $\operatorname{sn}^{2} x$, it follows that

$$
\phi_{i}^{1}(x)=\sqrt{\operatorname{dn} x+\operatorname{cn} x}\left[\operatorname{cn} x \alpha_{i}\left(\operatorname{sn}^{2} x\right)+\operatorname{dn} x \beta_{i}\left(\operatorname{sn}^{2} x\right)\right],
$$

where $\alpha_{i}(t)$ and $\beta_{i}(t)$ are polynomials in $t$ of degree $\frac{1}{2}(n-1)$ given by

$$
\begin{aligned}
& \alpha_{i}\left(\operatorname{sn}^{2} x\right)=\operatorname{dn}^{n-1} x \sum_{j=0}^{\frac{1}{2}(n-1)}\left\{\sigma_{i j} \tilde{T}_{n-2 j-1}(\operatorname{cd} x)+\rho_{i j} U_{n-2 j-1}(\operatorname{cd} x)\right\}, \\
& \beta_{i}\left(\operatorname{sn}^{2} x\right)=-\operatorname{dn}^{n-1} x \sum_{j=0}^{\frac{1}{2}(n-1)} \rho_{i j} U_{n-2 j-1}(\operatorname{cd} x) .
\end{aligned}
$$


Comparing (4.31) with Ref. [2] (p. 97), we see that $\phi_{i}^{1}$ is proportional to the non-meromorphic Lamé function $\mathrm{Ec}_{\ell}^{r_{i}+\frac{1}{2}}$,

$$
\phi_{i}^{1}(x) \propto \mathrm{Ec}_{\ell}^{r_{i}+\frac{1}{2}}, \quad 0 \leq i \leq n \equiv \ell-\frac{1}{2},
$$

where $r_{i}$ is the number of zeros of $\phi_{i}^{1}$ in the interval $(0,2 K)$. From Eq. (4.26) or, alternatively, from Eqs. (4.28)) and (4.31), we also obtain

$$
\phi_{i}^{2}(x)=\epsilon(x) \sqrt{\operatorname{dn} x-\operatorname{cn} x}\left[\operatorname{cn} x \alpha_{i}\left(\operatorname{sn}^{2} x\right)-\operatorname{dn} x \beta_{i}\left(\operatorname{sn}^{2} x\right)\right],
$$

so that

$$
\phi_{i}^{2}(x) \propto \mathrm{Es}_{\ell}^{r_{i}+\frac{1}{2}}, \quad 0 \leq i \leq n \equiv \ell-\frac{1}{2} .
$$

Similarly, when $n$ is even we rewrite Eq. (4.25) as follows:

$$
\begin{aligned}
& \phi_{i}^{1}(x)=\sqrt{\operatorname{dn} x+\operatorname{cn} x} \operatorname{dn}^{n} x {\left[p_{i, n / 2}+\sum_{j=0}^{\frac{n}{2}-1} \sigma_{i j} T_{n-2 j}(\operatorname{cd} x)\right.} \\
&\left.+\operatorname{cd} x(\operatorname{cd} x-1) \sum_{j=0}^{\frac{n}{2}-1} \rho_{i j} \tilde{U}_{n-2 j-2}(\operatorname{cd} x)\right] \\
&=\sqrt{\operatorname{dn} x+\operatorname{cn} x} \operatorname{dn}^{n-2} x\left[\operatorname{dn}^{2} x\left(p_{i, n / 2}+\sum_{j=0}^{\frac{n}{2}-1} \sigma_{i j} T_{n-2 j}(\operatorname{cd} x)\right)\right. \\
&\left.+\operatorname{cn} x(\operatorname{cn} x-\operatorname{dn} x) \sum_{j=0}^{\frac{n}{2}-1} \rho_{i j} \tilde{U}_{n-2 j-2}(\operatorname{cd} x)\right] .
\end{aligned}
$$

We thus obtain

$$
\phi_{i}^{1}(x)=\sqrt{\operatorname{dn} x+\operatorname{cn} x}\left[\alpha_{i}\left(\operatorname{sn}^{2} x\right)+\operatorname{cn} x \operatorname{dn} x \beta_{i}\left(\operatorname{sn}^{2} x\right)\right],
$$

where $\alpha_{i}(t)$ and $\beta_{i}(t)$ are polynomials in $t$ of respective degrees $\frac{n}{2}$ and $\frac{n}{2}-1$, defined by

$$
\begin{aligned}
\alpha_{i}\left(\operatorname{sn}^{2} x\right)= & \operatorname{dn}^{n} x\left[p_{i, n / 2}+\sum_{j=0}^{\frac{n}{2}-1} \sigma_{i j} T_{n-2 j}(\operatorname{cd} x)\right] \\
& +\operatorname{dn}^{n-2} x \operatorname{cn}^{2} x \sum_{j=0}^{\frac{n}{2}-1} \rho_{i j} \tilde{U}_{n-2 j-2}(\operatorname{cd} x), \\
\beta_{i}\left(\operatorname{sn}^{2} x\right)= & -\operatorname{dn}^{n-2} x \sum_{j=0}^{\frac{1}{2}(n-1)} \rho_{i j} \tilde{U}_{n-2 j-2}(\operatorname{cd} x) .
\end{aligned}
$$


From (4.28) we have

$$
\phi_{i}^{2}(x)=\epsilon(x) \sqrt{\operatorname{dn} x-\operatorname{cn} x}\left[\alpha_{i}\left(\operatorname{sn}^{2} x\right)-\operatorname{cn} x \operatorname{dn} x \beta_{i}\left(\operatorname{sn}^{2} x\right)\right] .
$$

Comparing, again, with Ref. [2] we deduce that Eqs. (4.34) and (4.36) also hold when $n$ is an even non-negative integer.

\subsection{Examples}

The case $n=0$, i.e, $\ell=1 / 2$, is particularly easy to deal with in the framework of our formalism. Indeed, in this case we can exactly compute one eigenvalue $E_{0}$ of the Lamé Hamiltonian, its corresponding two linearly independent eigenfunctions given by (4.38)-(4.41) with $n=0$ :

$$
\phi_{0}^{1}(x)=\sqrt{\operatorname{dn} x+\operatorname{cn} x}, \quad \phi_{0}^{2}(x)=\epsilon(x) \sqrt{\operatorname{dn} x-\operatorname{cn} x} .
$$

The eigenvalue $E_{0}$ is the root of the first degree polynomial $P_{1}$ or, equivalently, of $\hat{P}_{1}$ (since both polynomials differ by a constant factor, by (4.13)). From Eq. (3.17) with $j=0$ we get

$$
\hat{P}_{1}(E)=\left(E-b_{0}\right) \hat{P}_{0}=E-b_{0},
$$

and from (4.15) (with $n=0)$ it follows that

$$
E_{0}=b_{0}=\frac{1}{4}(1+m) \equiv \frac{1}{4}\left(1+k^{2}\right) .
$$

We have thus shown in a purely algebraic fashion that the general solution of the Lamé equation

$$
\psi^{\prime \prime}(x)+\frac{1}{4}\left(1+k^{2}-3 k^{2} \operatorname{sn}^{2} x\right) \psi(x)=0
$$

is a linear combination of the two functions (4.42).

Similarly, for $n=1$, i.e, $\ell=3 / 2$, the two algebraic eigenvalues of the Lamé Hamiltonian are the roots of the polynomial

$$
\hat{P}_{2}(E)=E^{2}-\frac{5}{2}(m+1) E+\frac{3}{16}\left(3 m^{2}+23 m+3\right)
$$

namely,

$$
\left.\begin{array}{l}
E_{0} \\
E_{1}
\end{array}\right\}=\frac{5}{4}(m+1) \mp \sqrt{m^{2}-m+1}
$$


Their associated eigenfunctions are easily computed using Eqs. (4.31)-(4.33), which for $n=1$ reduce to

$\alpha_{i}=2 p_{i 0}=2$,

$\beta_{i}=p_{i 1}-p_{i 0}=-P_{1}\left(E_{i}\right)-1=-\frac{2}{m} \hat{P}_{1}\left(E_{i}\right)-1=\frac{2}{m}\left[\frac{3}{4}(m+3)-E_{i}\right]-1$.

From Eq. (4.31) we obtain

$$
\left.\begin{array}{l}
\phi_{0}^{1} \\
\phi_{1}^{1}
\end{array}\right\}=\sqrt{\operatorname{dn} x+\operatorname{cn} x}\left[m \operatorname{cn} x+\left(1-m \pm \sqrt{m^{2}-m+1}\right) \operatorname{dn} x\right],
$$

where we have omitted a trivial constant factor. The remaining two eigenfunctions $\phi_{0}^{2}$ and $\phi_{1}^{2}$ can be easily obtained from (4.46) using (4.35):

$$
\left.\begin{array}{l}
\phi_{0}^{2} \\
\phi_{1}^{2}
\end{array}\right\}=\epsilon(x) \sqrt{\operatorname{dn} x-\operatorname{cn} x}\left[m \operatorname{cn} x-\left(1-m \pm \sqrt{m^{2}-m+1}\right) \operatorname{dn} x\right] .
$$

The latter formulas are easily seen to coincide with the ones at the end of Ref. [2].

As in the case of integer $\ell$, the computation of the non-meromorphic Lamé functions in closed form quickly becomes unmanageable as the degree of the critical polynomial $\hat{P}_{n+1}$ increases. For example, for $\ell=5 / 2$ (i.e., $n=2$ ) the algebraic eigenfunctions of the form (4.37) can be expressed as follows

$$
\begin{aligned}
\frac{\phi_{i}^{1}}{24 m^{2}} & =\sqrt{\operatorname{dn} x+\operatorname{cn} x}\left\{-25-130 m+87 m^{2}+(104+40 m) E_{i}-16 E_{i}^{2}\right. \\
& +m \operatorname{sn}^{2} x\left[25+34 m+9 m^{2}-(104+40 m) E_{i}+16 E_{i}^{2}\right] \\
& \left.+2 \operatorname{cn} x \operatorname{dn} x\left[25+430 m+21 m^{2}-8(13+11 m) E_{i}+16 E_{i}^{2}\right]\right\},
\end{aligned}
$$

where $E_{i}$ is one of the three real roots of the critical polynomial

$$
\begin{aligned}
\hat{P}_{3}(E)=E^{3}-\frac{35}{4}(m+1) E^{2}+ & \frac{7}{16}\left(37 m^{2}+138 m+37\right) E \\
& -\frac{5}{64}(m+1)\left(45 m^{2}+794 m+45\right) .
\end{aligned}
$$

The remaining three eigenfunctions $\phi_{i}^{2}(i=0,1,2)$ are obtained from the Eq. (4.48) using Eq. (4.41). When $m=1 / 2$, the energies $E_{i}$ can again be computed explicitly, i.e.,

$$
\left.\begin{array}{l}
E_{0} \\
E_{2}
\end{array}\right\}=\frac{35}{8} \mp \sqrt{7}, \quad E_{1}=\frac{35}{8}
$$


Consequently, Eq. (4.48) for the eigenfunctions yields the following explicit expressions:

$$
\begin{aligned}
& \left.\begin{array}{l}
-\frac{3}{4} \phi_{0}^{1} \\
-\frac{3}{4} \phi_{2}^{1}
\end{array}\right\}=\sqrt{\operatorname{dn} x+\operatorname{cn} x}\left[(5 \pm \sqrt{7}) \operatorname{sn}^{2} x-2(2 \pm \sqrt{7}) \operatorname{cn} x \operatorname{dn} x-7 \mp 2 \sqrt{7}\right] \\
& -\frac{1}{16} \phi_{1}^{1}=\sqrt{\operatorname{dn} x+\operatorname{cn} x}\left(\operatorname{sn}^{2} x+2 \operatorname{cn} x \operatorname{dn} x-\frac{7}{4}\right) .
\end{aligned}
$$

\section{References}

[1] Ince E L 1939-40 Proc. Roy. Soc. Edinb. 60 47-63

[2] Ince E L 1939-40 Proc. Roy. Soc. Edinb. 60 83-99

[3] Erdélyi A, Magnus W, Oberhettinger F and Tricomi F G 1955 Higher Transcendental Functions, vol. III (New York: Mc-Graw Hill)

[4] Arscott F M 1964 Periodic Differential Equations, (Oxford: Pergamon)

[5] Alhassid Y, Gürsey F and Iachello F 1983 Phys. Rev. Lett. 50 873-6

[6] Braibant S and Brihaye Y 1993 J. Math. Phys. 34 2107-14

[7] Kofman L, Linde A and Starobinsky A A 1994 Phys. Rev. Lett. 73 3195-8

[8] Greene P B, Kofman L, Linde A and Starobinsky A A 1997 Phys. Rev. D 56 6175-92

[9] Turbiner A V 1989 J. Phys. A: Math. Gen. 22 L1-L3

[10] González-López A, Kamran N and Olver P J 1994 Contemporary Mathematics 160 113-40

[11] Ushveridze A G 1994 Quasi-Exactly Solvable Models in Quantum Mechanics (Bristol: IOP)

[12] Olver P J 1997 GROUP21: Physical Applications and Mathematical Aspects of Geometry, Groups, and Algebras, ed H-D Doebner et al. (Singapore: World Scientific) 285-95

[13] Razavy M 1980 Am. J. Phys. 48 285-8 
[14] Finkel F, González-López A, and Rodríguez M A 1999 Preprint math$\mathrm{ph} / 9905020$

[15] Ward R S 1987 J. Phys. A: Math. Gen. 20 2679-83

[16] Ulyanov V V and Zaslavskii O B 1992 Phys. Rep. 216 180-251

[17] Brihaye Y and Godart M 1993 J. Math. Phys. 34 5283-91

[18] Turbiner A V 1988 Commun. Math. Phys. 118 467-74

[19] González-López A, Kamran N and Olver P J 1993 Commun. Math. Phys. 153 117-46

[20] Turbiner A V 1992 J. Phys. A: Math. Gen. 25 L1087-L93

[21] Finkel F and Kamran N 1998 Adv. Appl. Math. 20 300-22

[22] Turbiner A V and Ushveridze A G 1987 Phys. Lett. A126 181-3

[23] Finkel F, González-López A and Rodríguez M A 1996 J. Math. Phys. $373954-72$ 